\title{
Introdução à Programação e à Implementação de Processadores por Estudantes do Ensino Médio
}

\author{
Felipe Viel $^{3}$, André Raabe ${ }^{1,2}$, Cesar Zeferino ${ }^{1}$ \\ ${ }^{1}$ Mestrado em Computação Aplicada \\ ${ }^{2}$ Programa de Pós-graduação em Educação \\ ${ }^{3}$ Bacharelado de Engenharia da Computação \\ Universidade do Vale do Itajaí - Univali \\ Itajaí - SC - Brasil \\ \{viel, raabe, zeferino\}@univali.br
}

\begin{abstract}
This paper describes the initiation of high school students to computer programming, design of digital circuits and implementation of an educational purposes processor being an initiative to attract graduates to courses of Computer related areas, more specifically Computer Engineering. The project evaluated the use of BIP processor as an approach to foster computational thinking. The result pointed the program viability for small groups of students and the advantage of being supported by didactic resources used in first under graduation year.
\end{abstract}

Resumo. Este trabalho descreve a iniciação de estudantes de ensino médio a programação de computadores, projeto de circuitos digitais e implementação de um processador com fins educacionais, sendo uma iniciativa para captação de egressos do ensino médio para cursos na área da computação, mais especificamente na Engenharia de Computação. O projeto avaliou o uso do processador BIP como um aporte para fomentar o desenvolvimento do pensamento computacional. $O$ resultado apontou a viabilidade do programa proposto para pequenos grupos de estudantes e a vantagem de se apoiar nos recursos didáticos utilizados no primeiro ano dos cursos de graduação.

\section{Introdução}

A necessidade de profissionais nas áreas de Engenharia e Tecnologia têm crescido num ritmo exponencial em decorrência do uso cada vez maior de tecnologia na sociedade. Porém a formação destes profissionais não tem seguido o mesmo ritmo. Segundo Nascimento (2012), dados da Confederação Nacional de Indústrias (CNI) projetavam um déficit de 150 mil engenheiros no Brasil em 2012. O mesmo autor ainda menciona dados do Censo do Ensino Superior do Ministério da Educação (MEC) que mostravam que apenas $10 \%$ dos universitários estavam matriculados em carreiras ligadas à engenharia e tecnologia.

Aliado ao baixo ingresso de estudantes nestas áreas o alto índice de evasão dos cursos de graduação agravam a situação. Segundo Waltrick (2013), o Brasil deixou de colocar 60,3 mil engenheiros no mercado de trabalho em 2011 em função do abandono de estudantes matriculados em cursos de Engenharia. $\mathrm{Na}$ área de Tecnologia de Informação, de acordo com o IDC (EXAME, 2013), em 2015, o Brasil terá 117 mil 
vagas abertas para profissionais de tecnologia sem que os empregadores encontrem pessoal qualificado para atendê-las. Em toda a América Latina, até 2015, a procura por profissionais deve superar a oferta de mão de obra em $27 \%$.

Neste cenário, programas de fomento para estimular a atração de estudantes do ensino médio para cursos das áreas de Engenharia e Computação foram disparadas pelo Ministério da Ciência e Tecnologia (MCT). Em 2012, foi lançada a chamada CNPq/VALE S.A. No 05/2012 - Forma-Engenharia, com foco em cursos de Engenharia. Este artigo reporta a execução e os resultados obtidos com um projeto no escopo desta chamada, realizado pela Universidade do Vale do Itajaí (Univali) em parceria com o Colégio de Aplicação (CAU) da Universidade.

O projeto busca engajar os estudantes do ensino médio em atividades de introdução a programação e implementação de processadores buscando estimular a formação do pensamento computacional. Para isso apoia-se em uma abordagem interdisciplinar de ensino de Computação detalhada em Vieira (2013) a qual visa integrar o aprendizado de estudantes de graduação em diferentes disciplinas por meio de uma arquitetura simplificada de processador denominado BIP (Basic Instruction-Set Processor).

Com uso de uma IDE (Integrated Development Environment) denominada BipIDE os estudantes trabalham conceitos fundamentais sobre programação, operação e implementação de computadores. A experiência adquirida na pesquisa de Vieira (2013) motivou a proposta de estender a abordagem a pequenos grupos de estudantes do ensino médio na forma de um programa de atividades para introdução ao pensamento computacional.

O artigo está organizado da seguinte forma. Na seção 2 são descritos o processador BIP e o BipIDE, na seção 3 são detalhadas as atividades do programa de introdução ao pensamento computacional conduzido, na seção 4 são apresentados os resultados obtidos e na seção 5 são apresentadas as conclusões do artigo.

\section{BIP e BipIDE}

A especificação do processador BIP seguiu uma abordagem interdisciplinar envolvendo professores das áreas de Arquitetura de Computadores e de Programação de Computadores e buscando também promover o seu uso em diferentes disciplinas de cursos da área de Computação.

A especificação do BIP utilizou como premissas básicas: (i) facilitar o aprendizado do estudante a respeito do seu funcionamento e das relações ilustradas pelo processador; e (ii) viabilizar seu uso em outras disciplinas do curso. Em outras palavras, o processador deveria ser o mais simples possível para que um estudante calouro de um curso de graduação em Computação pudesse aprender o seu funcionamento e estivesse apto a programá-los nas primeiras semanas de aula de uma disciplina de introdução à programação. Ao mesmo tempo, o processador deveria ser completo o suficiente (ou seja, não hipotético) para poder ser utilizado como primeiro exemplo de processador em outras disciplinas de um curso da área de Computação, tais como: Circuitos Digitais, Arquitetura e Organização de Computadores e Compiladores, entre outras. Seguindo essas premissas, foi definida uma família de processadores com modelos incrementais 
com diferentes níveis de complexidade (BIP I, BIP II, BIP III e BIP IV). Cada modelo acrescenta novas instruções e suportes aos modelos anteriores (ZEFERINO et al, 2012).

Para promover a aprendizagem de programação de forma integrada a compreensão de como funciona o processador foi implementado um ambiente de desenvolvimento integrado denominado BipIDE. O BipIDE permite que o estudante escreva um programa em linguagem de alto nível (Portugol), visualize o código equivalente na linguagem de montagem do BIP e a execução das instruções, passo-apasso, nos códigos fonte e na organização do processador. O estudante também pode programar o processador diretamente na linguagem de montagem. O BipIDE foi desenvolvido no âmbito de um trabalho de conclusão de curso em Ciência da Computação (VIEIRA, 2009) que foi premiado com o primeiro lugar no Concurso de Teses, Dissertações e Trabalhos de Conclusão de Curso em Informática na Educação (CTD-IE) no SBIE 2009.

O processador BIP é utilizado nos Curso de Ciência e Engenharia de Computação da Univali no apoio ao ensino em diferentes disciplinas. Dentre as disciplinas que utilizam o BIP, podem ser destacadas as seguintes: Introdução à Programação ( $1^{\circ}$ período); Circuitos Digitais ( $2^{\circ}$ período); Arquitetura e Organização de Computadores ( $3^{\circ}$ período); e Compiladores ( $7^{\circ}$ período) (ZEFERINO et al., 2012; PEREIRA et al., 2012).

\section{O Programa de Introdução ao Pensamento Computacional}

À exemplo de outros programas de introdução ao pensamento computacional como Andrade (2013) e França e Amaral (2013), o propósito deste programa está em proporcionar aos estudantes vivências que possibilitem compreender e desenvolver conceitos relacionados ao cotidiano do estudo em computação. São muitas as possibilidades para efetivar este propósito, neste sentido serão detalhados os aspectos instrucionais, organizacionais e operacionais do programa que foi conduzido a fim de compartilhar a experiência vivenciada.

\subsection{Equipe Executora}

O Programa Forma-Engenharia foi proposto visando apoiar projetos com prazo máximo de execução de 15 meses envolvendo um grupo composto de professores de graduação, professor de ensino médio, estudante de graduação e de 2 a 4 estudantes de ensino médio.

No projeto conduzido na Univali foram solicitadas e contempladas uma bolsa ATP-B (Apoio Técnico em Extensão no País) para o professor de ensino médio, uma bolsa ITI-A (Iniciação Tecnológica e Industrial) para o estudante de graduação e quatro bolsas ITI-B para estudantes de ensino médio. A proposta também foi contemplada com recursos de custeio e de capital.

A equipe executora foi então composta dos seguintes integrantes:

- 2 professores de graduação: responsáveis pelo planejamento e supervisão das atividades realizadas pelos estudantes de graduação e de ensino médio; 
- 1 professor de ensino médio: responsável por relacionar as atividades realizadas no projeto com conteúdo da escolar e acompanhar o desenvolvimento dos estudantes de ensino médio;

- 1 estudante de graduação: responsável pelo capacitação dos estudantes e acompanhamento de suas atividades, sob a supervisão dos pesquisadores; e

- 4 estudantes de ensino médio: responsáveis por realizar os estudos e atividades práticas aplicadas pelo estudante de graduação, sob acompanhamento continuado dos professores de graduação e de ensino médio.

Para selecionar o bolsista ITI-A, foi feita uma divulgação junto aos estudantes de graduação por meio de lista de discussão. Dentre os candidatos interessados, foi selecionado estudante que apresentava o conhecimento técnico necessário e tinha experiência prévia como instrutor em curso de informática. Em sua entrevista, demonstrou ter a articulação necessária para atuar como monitor e instrutor do projeto.

Para selecionar os bolsistas ITI-B, foi feita uma palestra de divulgação a todos os estudantes do $1^{\circ}$ ano e do $2^{\circ}$ ano do Ensino Médio do Colégio de Aplicação (a chamada pública restringia as bolsas a estudantes dessas séries). Na palestra, foram apresentados os objetivos do Programa, a metodologia do projeto e detalhes adicionais sobre a sua operacionalização. Essas informações foram então encaminhadas em uma mensagem eletrônica enviada aos estudantes por meio de uma lista de discussão, abrindo oportunidade para que eles se aplicassem no processo de seleção de bolsistas para o projeto. De um universo de 150 estudantes, aplicaram-se apenas 4 candidatos (2,6\%), evidenciando a dificuldade de se atrair estudantes para a área. Como esses estudantes cumpriam o requisito do programa quanto ao bom desempenho escolar nas disciplinas de Ciências e Matemática no $1^{\circ}$ e no Ensino Médio, ambos foram selecionados.

\subsection{Plano Instrucional}

Após a seleção dos estudantes de ensino médio, foi planejado um programa de atividades com base na disponibilidade deles para as atividades do projeto. Visando não trazer prejuízo ao desempenho escolar dos estudantes e, ao mesmo tempo, mantê-los motivados com o projeto, todas as atividades foram programadas para serem realizadas em encontros em laboratório. Os encontros envolveram aulas expositivas, com aplicação prática imediata na forma de exercícios práticos em computador. A seguir são detalhadas a carga horária, as ferramentas utilizadas e as atividades desenvolvidas.

\subsubsection{Carga horária}

Inicialmente, foi estabelecido que o projeto iria ser realizado com 5 encontros semanais de 2 horas cada (10 horas por semana). Após, observou-se que os estudantes precisavam de tardes livres para outras atividades extracurriculares e o programa de capacitação foi revisto para 3 encontros semanais de 3 horas cada ( 9 horas por semana). Em períodos de provas concentradas, os estudantes foram dispensados para se dedicarem aos estudos necessários. No total, considerando apenas o ano de 2012, foram ministradas pelo menos 300 horas de aula em laboratório.

\subsubsection{Ferramentas utilizadas}


Diversas ferramentas foram utilizadas ao longo do desenvolvimento do projeto, incluindo as seguintes:

- IDE Bipide: ambiente de desenvolvimento educacional que permite relacionar os conceitos de lógica de programação com o hardware dos processadores BIP. Esse ambiente possibilita a criação de algoritmos e sua simulação na organização dos processadores BIP (VIEIRA, RAABE e ZEFERINO, 2010);

- IDE Portugol Studio: ambiente de desenvolvimento, com suporte à linguagem Portugol, tendo um intuito didático, voltado a iniciantes na área de programação; (NOSCHANG, et al., 2014)

- IDE CodeBlocks: ambiente de desenvolvimento com foco nas linguagens de alto nível C, C++, Fortran;

- IDE Altera Quartus II Web Edition: ambiente de desenvolvimento para projeto e síntese de circuitos digitais em FPGAs da Altera;

- Simulador ModelSim Altera Edition Software: ambiente de simulação para verificação do projeto de circuitos digitais; e

- Kits de desenvolvimento Mercúrio IV: placas para prototipação de circuitos digitais em FPGA.

\subsubsection{Atividades e Conteúdos Abordados}

As atividades práticas aplicadas aos estudantes foram organizados cinco etapas. Em cada uma delas são indicados os conteúdos abordados, conforme segue:

\section{Etapa 1 - Algoritmos e Programação (03-05/2013)}

Esta etapa envolveu a formação dos estudantes de ensino médio quanto a técnicas de programação de computadores. Inicialmente, foram apresentados conceitos fundamentais sobre o funcionamento de um computador (componentes e funções). Após, foram introduzidos conceitos básicos de lógica de programação. Nesta fase, os estudantes projetaram algoritmos para solução de problemas de matemática e de física, nesta etapa a participação do professor do ensino médio foi fundamental. Utilizaram também jogos de lógica e de programação como por exemplo Light Bot.

$\mathrm{Na}$ sequencia os estudantes aprenderam os fundamentos de programação com o uso da linguagem Portugol e uso do Ambiente BipIDE. O uso do BipIDE possibilitou além da resolução de algoritmos simples a compreensão do assembly correspondente e de conceitos de arquitetura e organização que seriam importantes nas etapas subsequentes. Visando estimular a aprendizagem fomentou-se a participação dos estudantes nas Olimpíadas Brasileiras de Informática - OBI 2013, para isso os estudantes foram introduzidos à linguagem $\mathrm{C}++$. Três bolsistas participaram da OBI (estudantes A, B e C), sendo que um deles avançou à segunda fase. O conteúdo trabalhado nesta etapa (com os meses de execução) incluiu:

- Conceitos sobre Computadores (03/2013): Organização básica de um computador; Memórias RAM, ROM; Processadores; Placa mãe; Periféricos. 
- Algoritmos e Programação (03/2013): Conceitos de Programação; Exercícios; Uso de jogos para estimular o raciocínio lógico (LigthBot).

- Programação na linguagem Portugol (03-04/2013): Uso dos ambientes de programação BipIDE e Portugol Studio; O que é linguagem de programação; Estrutura básica de um programa estruturado; Tipos de variáveis: Operadores lógicos, aritméticos e relacionais; Entrada, Processamento e saída; Desvios condicionais, laços de repetição; Vetores e Matrizes; Procedimentos; e

- Programação na linguagem C++ (04-05/2013): Uso do IDE CodeBlocks; Criação de um Projeto; Programação estruturada em C++; Variáveis, estruturas de condição, laços de repetição, operadores lógicos, aritméticos, relacionais, vetores, matrizes em $\mathrm{C}++$; Tradução de códigos e Portugol para $\mathrm{C}++$; Conceitos básicos de função e procedimento em $\mathrm{C}++$.

\section{Etapa 2 - Desenvolvimento de Aplicações (05-06/2013)}

Esta etapa envolveu o desenvolvimento e a simulação de aplicações para o BIP utilizando o ambiente Bipide, bem como aplicações baseadas na linguagem Portugol usando o Portugol Studio e na linguagem C++ usando o ambiente CodeBlocks. Dentre as aplicações desenvolvidas, podem ser citadas: (i) Calculadora com as quatro operações básicas; (ii) Animações usando caracteres; e (iii) Conversor de base de numeração (após o estudo desse conteúdo na Etapa 3);

\section{Etapa 3 - Capacitação em Eletrônica Digital (05-11/2013)}

Englobou a formação dos estudantes de ensino médio quanto a técnicas de projeto de circuitos digitais. Os estudantes realizaram estudos sobre sistemas de numeração, eletricidade, álgebra Booleana, portas lógicas, circuitos combinacionais, e circuitos sequenciais. Os estudos foram complementados por atividades práticas baseadas no uso de ferramenta de captura de esquemático e de simulação de circuitos digitais. O conteúdo trabalhado nesta etapa (com os meses de execução) incluiu:

- Sistemas de numeração (05/2013): Sistemas binário e hexadecimal; Conversão entre os sistemas de numeração binário, decimal e hexadecimal; Teoria dos conjuntos;

- Conceitos básicos sobre eletricidade (06/2013): Definição de eletricidade; Circuitos elétricos; Geração de eletricidade; Dispositivos semicondutores; Aplicações.

- Circuitos Digitais (06-11/2013): Conceitos de chave aberta e fechada; Expressões booleanas; Portas lógicas; Álgebra booleana; Tabela-verdade; Mapa de Karnaugh; Ambientes Quartus II e ModelSim; Circuitos digitais combinacionais: multiplexadores, demultiplexadores, codificadores, decodificadores, somadores, subtratores, somador/subtrator; Projeto e simulação de circuitos combinacionais; Latch e Flip-Flop; Circuitos sequenciais: registradores e contadores; Elaboração, construção e simulação de circuitos sequenciais; Implementação em FPGA dos exercícios.

Etapa 4 - Implementação e Verificação do BIP (11-12/2013) 
Envolveu o estudo da arquitetura e da organização do processador BIP I, a implementação dos seus componentes e do processador utilizando ferramenta de captura de esquemático do ambiente Quartus II, seguida da verificação do funcionamento correto dos componentes e do processador utilizando o simulador ModelSim.

\section{Etapa 5 - Prototipação de Aplicações sobre o BIP em FPGA (01-02/2014)}

Englobou a síntese do processador BIP em dispositivo lógico programável do tipo FPGA e a construção de protótipos de aplicações básicas de sistemas embarcados utilizando kits de desenvolvimento para FPGA e componentes eletrônicos adicionais. Foi desenvolvida uma aplicação de teste, na qual são utilizadas chaves para entrada de dados e LEDs para saída e depuração. A aplicação consiste em uma calculadora que lê os dados do teclado e realiza uma operação, apresentando os resultados nos LEDs.

\section{Resultados}

Como o foco do projeto esteve voltado para atração de jovens para as áreas de Engenharia e Computação, os resultados buscam descrever os avanços dos estudantes na direção de compreenderem conceitos fundamentais destas áreas e também a aquisição de competências relacionadas como o trabalho em grupo e a resolução de problemas.

O grupo de estudantes tinha quatro meninos (referidos a seguir como estudantes A, B,C e D), sendo três do $1^{\circ}$ ano do Ensino Médio e um deles no $2^{\circ}$ ano. A avaliação adotou um caráter formativo, sendo realizada no decorrer dos encontros uma vez que não foi adotado o formato de aula expositiva e sim de grupo de trabalho integrado. Em momentos específicos, foram realizadas avaliações formais (provas e trabalhos) referentes aos conteúdos e processos abordados.

A primeira avaliação compreendeu uma prova e um trabalho prático sobre Algoritmos e Programação. E a segunda avaliação também teve uma prova e um trabalho mas com o tema de Circuitos Digitais. Tanto as provas quanto os trabalhos foram contruídos em conjunto com os docentes e tiveram níveis de exigência similares aos aplicados nas disciplinas equivalentes do primeiro ano do curso de graduação em Ciência da Computação.

O trabalho aplicado em Algoritmos e Programação foi uma questão da Olimpíada Brasileira de Programação (OBI) de 2012 denominado quadrado mágico. O trabalho prático de Circuitos Digitais foia a implementação de um Cronometro Digital utilizando FPGA. As provas foi realizadas individualmente pelos estudantes enquanto que os trabalhos foi realizado em duplas. Os resultados das avaliações são apresentados na Tabela 1.

Tabela 1 - Resultados das Avaliações

\begin{tabular}{cccccc}
\hline \multirow{2}{*}{ Estudante (Ano E.M.) } & \multicolumn{2}{c}{ Algoritmos e Programação } & & \multicolumn{2}{c}{ Circuitos Digitais } \\
\cline { 2 - 3 } \cline { 5 - 6 } & Prova & Trabalho & & Prova & Trabalho \\
\hline A ( $2^{\circ}$ ano $)$ & 8,00 & 9,00 & & 8,10 & 10,00 \\
B (1 $1^{\circ}$ ano $)$ & 8,90 & 8,00 & & 8,00 & 10,00 \\
C (1 $1^{\circ}$ ano $)$ & 4,75 & 9,00 & & 4,30 & 10,00 \\
D (1 $1^{\circ}$ ano $)$ & 4,85 & 7,00 & & 4,50 & 10,00 \\
\hline
\end{tabular}


Ao analisar os resultados é possível perceber que mesmo com nível de exigência equivalente ao da graduação os estudantes A e B tiveram desempenhos satisfatórios. Os estudantes $\mathrm{C}$ e $\mathrm{D}$ demonstraram mais afinididade com as atividades práticas e nas provas não se sentiram motivados a estudar para estas, assim como fizeram os estudantes A e B. Em parte isso decorre de o projeto ser de cunho complementar e opcional para estes estudantes. Ainda é possível verificar que todos os estudantes tiveram um bom desempenho nas atividades práticas.

Além das avaliações formais as trajetórias dos estudantes foram continuamente monitoradas e registradas a partir da observação participante. A seguir uma síntese destes registros são apresentados a seguir.

Analisando o grupo como um todo foi possível perceber que no início os estudantes não tinham noções claras sobre o conceitos e a atuação do profissonal da área de Computação e apresentavam pouco interesse em ingressar em cursos nessa área. Após um ano de participação no projeto eles passaram a se identificar mais com a área da Computação bem como assuntos ligados a ciência e engenharia. Adquiriram conhecimento sobre lógica de de programação, descrição de hardware, projeto e síntese em FPGA, circuitos digitais, uso de ferramentas de projeto e simulação de sistemas digitais e construiram conhecimentos sobre sistemas embarcados.

Os estudantes, com auxílio do professor do ensino médio que participou do projeto passaram a utilizar o conhecimento sobre programação para resolver e automatizar a resolução de problemas de Física e Matemática nas correspondentes disciplinas do ensino médio. Os estudantes A, B e C participaram da fase regional da OBI em 2013 sendo que o estudante B classificou-se para fase estadual. Três dos quatro estudantes passaram a demonstrar interesse em ingressar em cursos da área de computação conforme elucida o relato das trajetórias individuais a seguir.

No início do projeto o aluno A mostrou-se empolgado e motivado pois o projeto. Ele demonstrou facilidade de aprendizagem em especial em assuntos ligados ao hardware e teve um comportamento concentrado e estudioso ao longo do projeto. Melhorou sua capacidade de se expressar. Foi o primeiro a manifestar o interesse em ingressar em um curso superior na área da computação, ao que tudo indica será a Engenharia de Computação.

$\mathrm{O}$ aluno B dentre os alunos que ingressaram no projeto foi o que apresentou uma aprendizagem mais rápida da lógica de programação e circuitos digitais. Se mostrou mais interessado e identificado com a área de software. Aprendeu a trabalhar em grupo (que era uma dificuldade no início) e passou a expressar suas ideias junto aos colegas. Manifestou interesse em ingressar em Ciência da Computação.

$\mathrm{O}$ aluno $\mathrm{C}$, que dentre os três alunos era o que apresentava maior envolvimento prévio com a área de engenharia (devido ao histórico familiar), estando assim um pouco mais familiarizado com assuntos abordados. Mostrou-se mais motivado com trabalhos envolvendo hardware, como sistemas embarcados e atividades práticas. Melhorou em aspectos que apresentava dificuldades como em manter o foco durante estudo e em expressar suas dificuldades. Desenvolveu um espírito de liderança dentro do grupo. Manifestou afinidade com a área de Engenharia ainda indefinido em relação a especialidade entre mecânica, aeroespacial ou computação. 
$\mathrm{O}$ aluno $\mathrm{D}$ foi o que necessitou se empenhar mais por ter ingressado mais tarde que os demais participantes do projeto (isso o motivou no início). Se mostrou bastante empenhado, mesmo tendo mais dificuldade em dominar os assuntos abordados. 'Com o passar do projeto tornou-se dispersivo e dependente dos colegas. Apresentou mais interesse nos temas relacionados a software e tornou-se mais comunicativo ao longo da convivência com o grupo. Passou a explorar por conta própria engines de jogos. Até o momento não manifestou interesse de ingressar em Computação e Engenharias.

Destaca-se que o formato de atividades práticas integradas as explicações de conceitos auxiliou a manter os estudantes motivados durante o projeto. Os resultados obtidos no primeiro ano justificaram uma solicitação de prorrogação deste. Esta solicitação foi atendida pelo CNPq e no ano corrente (2014), os estudantes estão seguem aprendendo novas técnicas e tecnologias para implementação de sistemas computacionais.

\section{Conclusões}

O presente trabalho apresentou um programa de introdução ao pensamento computacional que proporcionou uma introdução a programação de computares e projeto de circuitos digitais com foco na implementação do processador BIP. Diferente de outras abordagens de introdução ao pensamento computacional que geralmente abordam mais estudantes e usam jogos e ambientes com propostas lúdicas (ex: Scratch) esta proposta foi fortemente atrelada aos conteúdos existentes nos cursos de Ciência e Engenharia da Computação e buscou utilizar as ferramentas usadas nestes cursos.

O desempenho dos estudantes nas avaliações e o cumprimento das metas do projeto dão indicativos de que esta abordagem é viável, pelo menos em pequenos grupos. Considera-se ainda que a abordagem apresenta um aspecto vantajoso de poder se valer dos conhecimentos, infraestrutura e materiais disponíveis nos cursos de graduação para sua efetivação. Por exemplo, todas as ferramentas utilizadas são de uso comum nas disciplinas dos primeiro ano, as avaliações utilizadas foram semelhantes as utilizadas, o estudante de graduação que atuou como tutor dos estudantes de ensino médio pode em diversos momentos abordar os professores das disciplinas correlatas a fim de esclarecer dúvidas e estes compartilharam planos de aula e sugestões de atividades para que este aplicasse com os estudantes.

Cumpre salientar que o programa CNPq/VALE S.A. No 05/2012 - FormaEngenharia a qual fomenta este projeto deixa abertura para que sejam propostas diferentes configurações de projetos a fim de atrair talentos para as áreas e Engenharia e Computação. Neste sentido considera-se que o relato de experiência apresentado neste artigo pode contribuir para discussão acerca de modelos instrucionais que podem ser aplicados o escopo deste programa de fomento.

O projeto, mesmo tendo cumprido suas metas teve sua continuidade assegurada pela Universidade tendo em vista o interesse dos estudantes em prosseguirem evoluindo seu nível de conhecimento na direção de um aprofundamento em assuntos mais complexos, como a utilização de VHDL para melhoria do projeto das versões II e III do processador BIP. 


\section{Referencias}

ANDRADE, D. et al. Proposta de Atividades para o Desenvolvimento do Pensamento Computacional no Ensino Fundamental. Anais do Workshop de Informática na Escola, Congresso Brasileiro de Informática na Educação, 2013.

EXAME. Falta de profissionais de TI se agravará no Brasil, diz IDC. São Paulo: Abril, 2013. Disponível em: <http://exame.abril.com.br/carreira/noticias/falta-de-profissionais-de-ti-seagravara-no-brasil-diz-idc>. Acesso em: 4 jul. 2014.

FRANÇA, Rozelma; AMARAL, Haroldo. Proposta Metodológica de Ensino e Avaliação para o Desenvolvimento do Pensamento Computacional com o Uso do Scratch. Anais do Workshop de Informática na Escola, Congresso Brasileiro de Informática na Educação, 2013.

MORANDI, Diana; PEREIRA, Maicon Carlos; RAABE, André Luis Alice; ZEFERINO, Cesar Albenes . Um processador básico para o ensino de conceitos de arquitetura e organização de computadores. Hífen, Uruguaiana, v. 30, p. 73-80, 2006.

NASCIMENTO, Cristiane. Brasil tem déficit de 150 mil engenheiros: mesmo com uma das maiores médias salariais, carreira atrai só $10 \%$ dos universitários. Rio de Janeiro: O Globo, 2012. Disponível em: <http://oglobo.globo.com/economia/brasil-tem-deficit-de-150-milengenheiros-6474231>. Acesso em: 4 jul. 2014.

NOSCHANG, Luis Fernando; PELZ, Fillipi; DE JESUS, Elieser A.; RAABE, André. Portugol Studio: Uma IDE para Iniciantes em Programação. Workshop de Educação em Computação, Anais do Congresso Anual da Sociedade Brasileira de Computação, 2014.

PEREIRA, M. C.; VIEIRA, P. V; RAABE, A. L. A. e ZEFERINO, C. A. A basic processor for teaching digital circuits and systems design with FPGA. In: SOUTHERN PROGRAMMABLE LOGIC CONFERENCE, 8., Bento Gonçalves, 2012. Proceedings... Pelotas: UFPel, 2012.

VIEIRA, Paulo Viniccius. Bipide: Ambiente de Desenvolvimento Integrado para Utilização dos Processadores BIP no Ensino de Programação. Trabalho de Conclusão de Curso. Graduação em Ciência da Computação - Universidade do Vale do Itajaí, 2009.

VIEIRA, P. V; RAABE, A. L. A. e ZEFERINO, C. A. Bipide: ambiente de desenvolvimento integrado para a arquitetura dos processadores BIP. Revista Brasileira de Informática na Educação, v. 18, n. 1, 2010. ISSN 1414-5685

VIEIRA, P. V. Avaliação Empírica da Proposta Interdisciplinar de uso dos Processadores BIP. Dissertação de Mestrado em Computação Aplicada, Universidade do Vale do Itajaí, 2013.

WALTRICK, Rafael. Alta taxa de desistência na universidade causa déficit de engenheiros. Londrina: Gazeta do Povo, 2013. Disponível em: $<$ http://www.gazetadopovo.com.br/vidaecidadania/conteudo.phtml?id=1405796\&tit=Altataxa-de-desistencia-na-universidade-causa-deficit-de-engenheiros $>$. Acesso em: 4 jul. 2014.

ZEFERINO, Cesar Albenes; RAABE, André L.A.; VIEIRA, P. V.; PEREIRA, Maicon Carlos. Um enfoque interdisciplinar no ensino de Arquitetura de Computadores. In: MARTINS, Carlos Augusto Paiva da Silva; NAVAUX, Philippe Olivier Alexandre; AZEVEDO, Rodolfo Jardim de; KOFUJI, Sérgio Takeo (Org.). Arquitetura de Computadores: educação, ensino e aprendizado. 1ed. Porto Alegre: Sociedade Brasileira de Computação (SBC), 2012, v. , p. 165-193. 